\title{
新型槲皮素缀合物的合成与降糖活性研究
}

\begin{tabular}{|c|c|c|c|c|c|c|}
\hline 潘国军 $a$ & 肖 & 娜 $b$ & 沈 & 棣 ${ }^{a}$ & 陈明朗 ${ }^{a}$ & 李 \\
\hline 芦 & 逵 ${ }^{a}$ & 杨 & 杨 ${ }^{a}$ & 孟 & 欣 $*, a$ 郁 & 彭*,a \\
\hline
\end{tabular}

\begin{abstract}
摘要 经过五步反应以 $15 \% \sim 46 \%$ 的总收率成功合成了 8 个新型的槲皮素缀合物 $\mathbf{2 0} \sim \mathbf{2 7}$. 以芦丁为起始原料, 经 $O$-苄 基化、酸催化糖苷水解、烷基化等步骤, 获得重要中间体 5,7,3',4'四苄氧基-3- $O$-炔丙基檞皮素; 再经过 Click 反应, 得

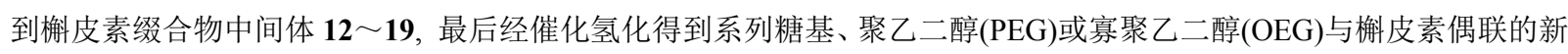
型缀合物. 经体外 3T3-L1 脂肪细胞水平降糖活性测试, 结果显示缀合物 $\mathbf{2 4 , 2 6}$ 和 27 具有较好的降糖活性. 关键词＼cjkstart触皮素缀合物; Click 反应; 合成; 降糖活性; 脂肪细胞
\end{abstract}

\section{Synthesis and Hypoglycemic Activity of Novel Quercetin Conjugates}

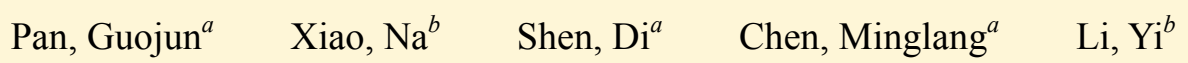 \\ Lu, Kui ${ }^{a} \quad$ Yang, Yang ${ }^{a} \quad$ Meng, Xin ${ }^{*, a} \quad$ Yu, Peng ${ }^{*, a}$ \\ $\left({ }^{a}\right.$ College of Technology, Tianjin University of Science and Technology, Tianjin 300457) \\ $\left({ }^{b}\right.$ College of Traditional Chinese Medicine, China Pharmaceutical University, Nanjing 210000)
}

\begin{abstract}
Eight novel quercetin conjugates $\mathbf{2 0} \sim 27$ were successfully synthesized through a concise procedure with $15 \% \sim$ $46 \%$ overall yields by using Rutin as the starting material. After $O$-benzylation, glycoside hydrolysis and $O$-propargylation, rutin was converted to the key intermediate 5,7,3',4'-tetrabenzyloxy-3-O-propargylquercetin, which subsequently 'clicked' with azido containing glycosides, poly(ethylene glycol) (PEG) or oligo(ethylene glycol) (OEG) to provide the fully protected quercetin conjugates 12 $\sim 19$. After global deprotection, the resulting quercetin conjugates were evaluated on hypoglycemic activities at 3T3-L1 adipocyte level. The results showed that conjugates 24, 26 and 27 exhibited good hypoglycemic effects.
\end{abstract}

Keywords quercetin conjugate; click reaction; synthesis; hypoglycemic activity; adipocyte

槲皮素(3,3',4',5,7-五羟基黄酮)属于黄酮醇类化合 物, 是一种天然存在的小分子植物次生代谢产物 ${ }^{[1 \sim 3]}$. 它主要存在于日常食用的各种蔬菜、水果、茶以及葡萄 酒中 ${ }^{[4]}$, 并具有抗心血管疾病、抗氧化、降血糖、抗肿 瘤、抗炎、抗病毒等多种药理活性 ${ }^{[5 \sim 14]}$. 另一方面, 葪 皮素等黄酮类化合物普遍存在水溶性差的问题，限制了 其应用范围，所以改善有药用价值的化合物的水溶性问 题显得至关重要.

值得一提的是, 水溶性单糖或二糖来源广泛, 可在 体内环境中顺利降解, 是一种绿色无害的药用材料. 例
如葡萄糖、半乳糖、甘露糖、乳糖等, 它们不仅为人体提 供能量, 还以糖苷的形式存在于各种组织和器官中, 如 大脑和神经组织等 ${ }^{[15]}$, 同时它们还是一些糖蛋白的重要 组成成分, 参与到各种生命活动过程中. 另外, 聚乙二醇 (PEG)或寡聚乙二醇 $(\mathrm{OEG})$ 具有良好的生物相容性和水溶 性，与药物配伍制剂可以改善药物的溶解性及靶向性等. 因此，本工作通过应用 “Click 反应” 将槲皮素与此类分 子偶联，旨在改善其水溶性，提高其生物利用度.

Click 反应是 2001 年诺贝尔化学奖获得者 Sharpless 提出的一种快速、高效、高选择性的合成方法. 此反应

* Corresponding authors. E-mail: mengxin@tust.edu.cn; yupeng@tust.edu.cn

Received August 2, 2016; revised September 5, 2016; published online September 12, 2016.

Project supported by the National Natural Science Foundation of China (Nos. 21502139, 21402140) and the Science and Technology Commissioner Foundation of Tianjin (No. 15JCTPJC56200).

国家自然科学基金(Nos. 21502139, 21402140)及天津市科技特派员(No. 15JCTPJC56200)资助项目. 
是利用 $\mathrm{Cu}(\mathrm{I})$ 催化叠氮化物与端基炔进行 1,3-偶极环加 成得到具有区域选择性的 1,4-二取代 1,2,3-三唑类化合 物 ${ }^{[15,16]}$. 本工作利用此反应为关键反应，在槲皮素结构 引入糖基、 $\mathrm{PEG}$ 或 $\mathrm{OEG}$, 获得了系列新型槲皮素衍生物
20２7 (Scheme 1)，并在 3T3-L1 脂肪细胞水平进行葡 萄糖消耗实验和苂光探针标记的 $2-D$-脱氧葡萄糖示踪

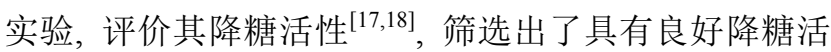
性的新型槲皮素衍生物.<smiles>[R10]COc1c(-c2ccc(O)c(O)c2)oc2cc(O)cc(O)c2c1=O</smiles><smiles>C#CCOc1c(-c2ccc(OCc3ccccc3)c(OCc3ccccc3)c2)oc2cc(OCc3ccccc3)ccc2c1=O</smiles>

$12 \sim 19$

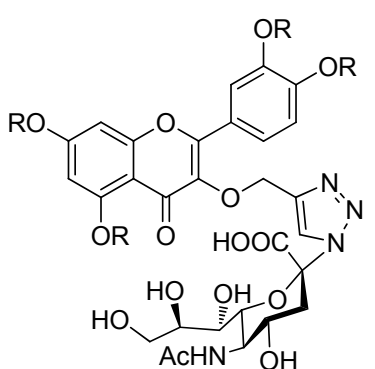

$12 \mathrm{R}=\mathrm{Bn}$ $20 \mathrm{R}=\mathrm{H}$

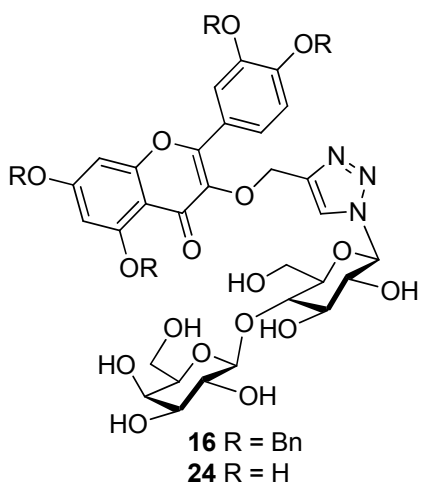

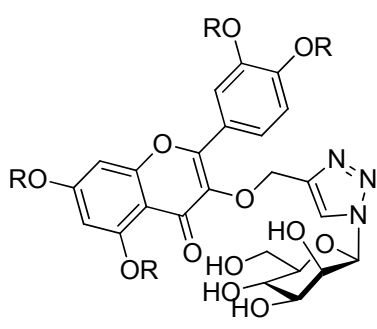

$13 \mathrm{R}=\mathrm{Bn}$ $21 \mathrm{R}=\mathrm{H}$

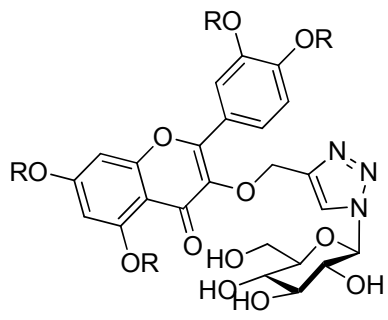

$14 \mathrm{R}=\mathrm{Bn}$

$22 \mathrm{R}=\mathrm{H}$

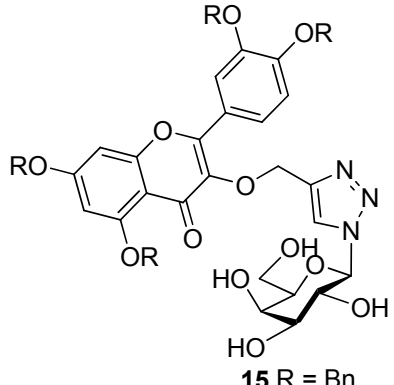

$15 \mathrm{R}=\mathrm{Bn}$

$23 \mathrm{R}=\mathrm{H}$<smiles>[R20]c1cc([R2])c2c(=O)c(OCc3cn(CCOCCOCCOCCO)nn3)c(-c3ccc([R2])c([R])c3)oc2c1</smiles><smiles>[R20]c1cc([R20])c2c(=O)c(OCc3cn(CCOC(C)COC(C)(C)C)nn3)c(-c3ccc([R20])c([R])c3)oc2c1</smiles>

Reagent and conditions: (a) $\mathrm{BnBr}, \mathrm{K}_{2} \mathrm{CO}_{3}$, DMF, reflux; (b) $\mathrm{HCl}$, EtOH, reflux; (c) 3-bromo-1-propyne, $\mathrm{K}_{2} \mathrm{CO}_{3}$, DMF, r.t.; (d) $4 \sim 11$ (Group- $\mathrm{N}_{3}$ ), $\mathrm{CuSO}_{4}$, ascorbic acid, THF/H $\mathrm{H}_{2} \mathrm{O}(V: V=1: 1) ;(e) \mathrm{H}_{2}, \mathrm{Pd} / \mathrm{C} 10 \%, \mathrm{MeOH}$

图式 1 化合物 $20 \sim 27$ 的合成路线

Scheme 1 Synthetic route of compounds $\mathbf{2 0} \sim \mathbf{2 7}$ 


\section{1 结果与讨论}

以来源丰富的芦丁为起始原料, 分别经过 $O$-苄基 化、酸性条件下水解糖苷键、 $O$-炔丙基化步骤, 合成得 到了重要中间体 3- $O$-炔丙基-5,7,3',4'-四芐氧基檞皮素 (3). 在苄基化保护时, 由于芦丁结构中羟基数量较多, 且反应活性不同, 一般反应条件较难实现全部芐基化. 因此我们对该步反应条件进行了优化: 先将芦丁溶于 $N, N$-二甲基甲酰胺(DMF), 加入碳酸钾, 升温至 $45{ }^{\circ} \mathrm{C}$, 搅拌 $0.5 \mathrm{~h}$, 再加入溴化茮, 密闭条件下反应 $1 \mathrm{~d}$. 收率大 大提升. 之后, 反应液经抽滤、减压蒸馏, 再加入盐酸/ 乙醇溶液 $(V: V=1: 10)$ 回流 $3 \mathrm{~h}$, 经中和、抽滤得到粗 产品 2 , 直接用于下一步反应, 得到重要的中间体 3, 这 三步反应的收率为 $53 \%$.

利用 “Click 反应” 将含有炔丙基的中间体 $\mathbf{3}$ 分别 与异头位叠氮基取代的半乳糖、甘露糖、乳糖、葡萄糖、 唾液酸等糖类分子偶联, 然后在 $10 \% \mathrm{Pd} / \mathrm{C}$ 条件下催化 氢化得到一系列新型檞皮素缀合物 $20 \sim 27$. 据文献[15] 报道, 在硫酸铜、抗坏血酸钠、 $\mathrm{TBAB}$ 、二氯甲烷与饱 和碳酸氢钠水溶液条件下, 此反应具有较高反应收率. 本工作在此基础上对反应条件进行了进一步优化, 即在 硫酸铜、抗坏血酸钠、四氢呋喃水溶液条件下反应 $8 \mathrm{~h}$, 反应收率高达 $80 \%$ ～95\%.

檞皮素缀合物 $20 \sim 27$ 均为未见文献报道的全新化 合物, 本工作对其进行了核磁共振氢谱( ${ }^{1} \mathrm{H}$ NMR)、核磁 共振碳谱 $\left({ }^{13} \mathrm{C} \mathrm{NMR}\right)$ 、高分辨质谱(HRMS)、MALDITOF-MS 及熔点测定.

以二甲双胍为阳性对照, 二甲基亚砜(DMSO)为溶 剂, 在 3T3-L1 脂肪细胞水平进行了体外糖消耗的生物 活性评价(实验结果见图 1A). 缀合物 20２7 与空白组 相比, 均显示较高的糖消耗作用; 但与檞皮素比较, 活 性没有显著性差异. 推断可能与檞皮素缀合物与䚞皮素 在 DMSO 溶剂中均具有良好的溶解性有关. 为了研究 水溶性差异对糖消耗作用的影响, 在实验中改用磷酸盐 缓冲液(PBS)对化合物进行溶解和稀释, 实验结果见图 1B. 结果显示䚞皮素在促进糖消耗方面有显著性下降, 推断此现象可能与檞皮素在 PBS 条件下溶解性较差有 关, 相比而言, 缀合物 24, 26, 27 在相同条件下则可以完 全溶解并保持较好的促进糖消耗的作用.

为了更直观地评价榆皮素缀合物的降糖活性, 进一 步用苂光探针标记的 2- $D$-脱氧葡萄糖苂光实验(苂光强 度越强, 促进糖消耗的能力越强) 评价其降糖活性, 实 验结果见图 2. 缀合物 24, 26, 27 荧光强度与空白组相 比, 苂光更强, 与阳性对照组相比, 强度相当, 此结果 进一步显示槲皮素缀合物确实有较好的降糖活性.

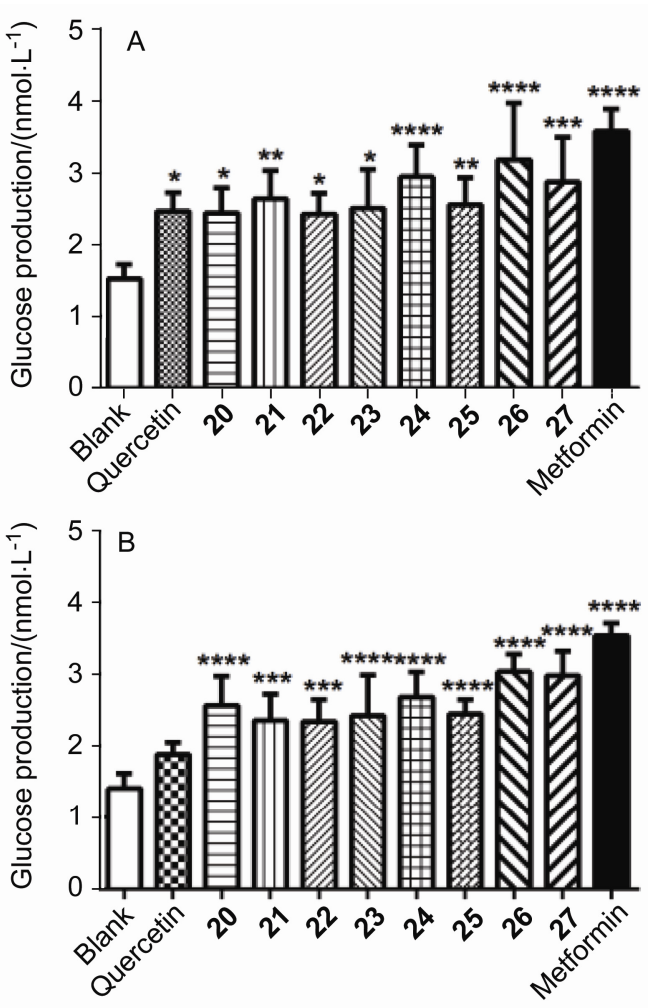

图 $120 \sim 27$ 对脂肪细胞糖消耗的作用

Figure $1 \quad 20 \sim 27$ promoted glucose consumption in adipocytes (A): Dissolved in DMSO and diluted with PBS. (B): Dissolved in PBS. ${ }^{*} p<0.05,{ }^{* *} p<0.01,{ }^{* * *} p<0.001,{ }^{* * * *} p<0.0001$ vs. blank

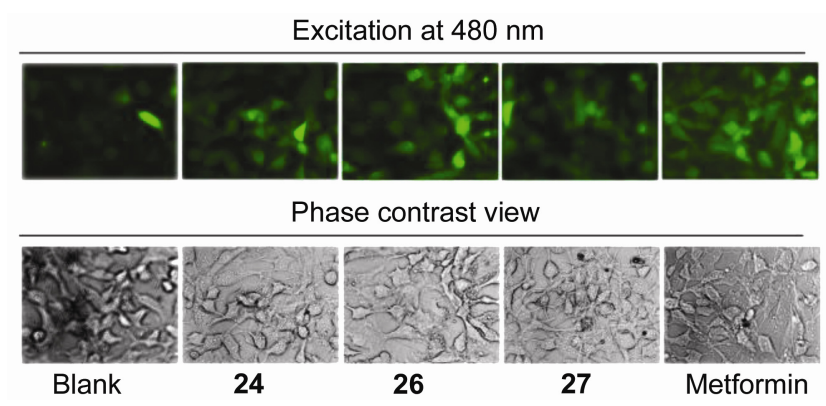

图 2 二甲双胍和缀合物 24, 26 和 27 对脂肪细胞 2-脱氧葡萄 糖摄取的作用

Figure 2 Metformin as well as conjugates 24, 26 and 27 regulated 2-NBDG uptake in adipocytes

These compounds were dissolved in PBS. 24, 26, $27\left(10 \mu \mathrm{mol} \cdot \mathrm{L}^{-1}\right)$, metformin $\left(1 \mathrm{mmol} \cdot \mathrm{L}^{-1}\right)$

\section{2 结论}

根据设计的合成路线，成功合成了 8 个新型檞皮素 缀合物 $20 \sim 27$. 目标化合物的结构经 ${ }^{1} \mathrm{H} N \mathrm{NMR},{ }^{13} \mathrm{C}$ NMR, HRMS 和 MALDI-TOF-MS 等确证. 该方法具有 合成路线简单、反应条件温和、收率高等优点. 细胞水 平降糖活性研究表明，葪皮素缀合物降糖作用的强弱与 其本身溶解性有直接关系, 水溶性越好, 降糖作用越强. 而且, 苂光探针标记的 2- $D$-脱氧葡萄糖苂光实验进一步 
验证了葪皮素缀合物具有较好的降糖效果.

\section{3 实验部分}

\section{1 仪器与试剂}

循环水式真空葲，河南省予华仪器有限公司; 集热 式恒温加热磁力搅拌器, 郑州长城科工贸; 低温恒温反 应浴，巩义市京华仪器有限公司; AVANCE III-400MHz 型核磁共振仪, 瑞士 Bruker 公司; 数字熔点仪 WRS-1B, 上海精密科学仪器有限公司. 柱层析硅胶(青岛海洋化 工厂产品). 所用试剂和溶剂为化学纯或分析纯, 要求 无水的溶剂均经除水和重蒸处理.

\section{2 化合物合成}

$\alpha$-叠氮唾液酸甲酯(4)参考文献[19]制备, $\beta$-叠氮甘 露糖 $(5) 、 \beta$-叠氮葡萄糖 $(6) 、 \beta$-叠氮半乳糖 $(7)$ 参考文献 $[20]$ 制备, $\beta$-叠氮乳糖 $(8)$ 参考文献[21]制备, 叠氮化三缩 四乙二醇(9)、叠氮化聚乙二醇单甲醚 2000 (10)参考文 献[22]制备，11-叠氮-3,6,9-三氧杂十一烷基-半乳糖苷 (11)参考文献[23]制备.

\subsubsection{5,7, 3', $4^{\prime}$-四苄氧基檞皮素(2)的合成}

芦丁 $(6.10 \mathrm{~g}, 10.00 \mathrm{mmol})$ 溶于 $100 \mathrm{~mL} N, N$-二甲基 甲酰胺(DMF)中, 加入无水碳酸钾 $(6.91 \mathrm{~g}, 50.00 \mathrm{mmol})$, $45{ }^{\circ} \mathrm{C}$ 搅拌 $30 \mathrm{~min}$ 后加入溴化茮溶液(8.55 g, 50.00 $\mathrm{mmoL}$ ), 继续搅拌 $48 \mathrm{~h}$, 经薄层色谱(TLC)检测反应完 全后, 过滤除去碳酸钾, 减压蒸馏除去溶剂, 粗产品直 接用于下一步反应. 加入 $300 \mathrm{~mL}$ 乙醇和 $30 \mathrm{~mL}$ 浓盐酸, 回流反应 $3 \mathrm{~h}$, 冷却至室温, 饱和碳酸氢钠水溶液中和 反应体系至中性, 抽滤, 洗涤滤饼, 干燥, 二氯甲烷重 结晶得黄色固体 $24.04 \mathrm{~g}$, 两步总产率 $61 \%$. 粗产品未 经分离纯化直接用于下一步反应.

3.2.23-炔丙氧基-5,7,3', $4^{\prime}$-四芳氧基斛皮素(3) 的合 成

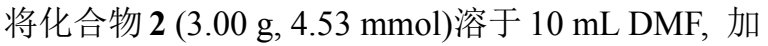
入无水碳酸钾 $(688 \mathrm{mg}, 4.98 \mathrm{mmol})$ 及溴丙炔 $(592 \mathrm{mg}$, $4.98 \mathrm{mmoL}$ ), 室温摚拌过夜, TLC 检测反应完全, 减压 旋蒸, 柱层析分离纯化 [洗脱剂: $V$ (石油醚) : $V$ (乙酸乙 酯) $=10 ： 1$ )]得白色固体 $32.76 \mathrm{~g}$, 产率 87\%. m.p. 302 $305{ }^{\circ} \mathrm{C} ;{ }^{1} \mathrm{H}$ NMR $\left(400 \mathrm{MHz}, \mathrm{CDCl}_{3}\right) \delta: 7.86(\mathrm{~d}, J=2.0$ $\mathrm{Hz}, 1 \mathrm{H}), 7.65$ (dd, $J=8.8 \mathrm{~Hz}, 2.0 \mathrm{~Hz}, 1 \mathrm{H}), 7.59$ (d, $J=7.6$ $\mathrm{Hz}, 2 \mathrm{H}), 7.50 \sim 7.28(\mathrm{~m}, 18 \mathrm{H}), 6.98(\mathrm{~d}, J=8.4 \mathrm{~Hz}, 1 \mathrm{H})$, $6.51(\mathrm{~d}, J=2.0 \mathrm{~Hz}, 1 \mathrm{H}), 6.42(\mathrm{~d}, J=2.0 \mathrm{~Hz}, 1 \mathrm{H}), 5.25$ (s, $2 \mathrm{H}), 5.21(\mathrm{~s}, 2 \mathrm{H}), 5.18(\mathrm{~s}, 2 \mathrm{H}), 5.06(\mathrm{~s}, 2 \mathrm{H}), 4.88(\mathrm{~d}, J=$ $2.4 \mathrm{~Hz}, 2 \mathrm{H}), 2.56(\mathrm{t}, J=2.4 \mathrm{~Hz}, 1 \mathrm{H}) ;{ }^{13} \mathrm{C} \mathrm{NMR}(101 \mathrm{MHz}$, $\left.\mathrm{CDCl}_{3}\right) \delta: 173.6,162.9,159.8,158.7,153.6,150.8,148.2$, $138.5,137.1,136.8,136.3,135.7,128.80,128.64,128.60$,
$128.55,128.48,128.0,127.9,127.7,127.6,127.3,127.2$, $126.6,123.9,122.4,115.5,113.7,109.9,98.1,93.9,79.2$, 75.8, 71.4, 70.9, 70.8, 70.5, 59.2; MS (ESI) $\mathrm{m} / z: 701.2$ $[\mathrm{M}+\mathrm{H}]^{+}$; HRMS (ESI) calcd for $\mathrm{C}_{46} \mathrm{H}_{37} \mathrm{O}_{7}[\mathrm{M}+\mathrm{H}]^{+}$ 701.2534, found 701.2531.

3.2.3 1- $[N-1-(\alpha-N-$ 乙酰神经氨酸甲酯 $)]-4-[3-O$ - 亚甲 基-(5,7,3',4'-四芐氧基槲皮素)]-[1,2,3]-三唑(12) 的合 成

称取化合物 3 (301 mg, $0.43 \mathrm{mmoL})$ 、化合物 4 (144 $\mathrm{mg}, 0.43 \mathrm{mmoL}) 、$ 硫酸铜 $(69 \mathrm{mg}, 0.43 \mathrm{mmoL})$ 及 $(+)-L-$ 抗坏血酸钠 $(85 \mathrm{mg}, 0.43 \mathrm{mmoL})$ 于 $25 \mathrm{~mL}$ 圆底烧瓶中, 加入四氢呋喃/水 $(V: V=1: 1) 10 \mathrm{~mL}$, 室温搅拌 $10 \mathrm{~h}$, 减压蒸馏除去溶剂, 柱层析分离纯化 [洗脱剂: $V($ 二氯甲 烷 $): V($ 甲醇 $)=30 ： 1)$ ]得黄色固体 $12320 \mathrm{mg}$, 产率 $72 \%$. m.p. $124.3 \sim 124.5{ }^{\circ} \mathrm{C} ;{ }^{1} \mathrm{H}$ NMR $(400 \mathrm{MHz}$, DMSO- $\left.d_{6}\right) \delta: 8.47(\mathrm{~s}, 1 \mathrm{H}), 7.99(\mathrm{~d}, \quad J=8.0 \mathrm{~Hz}, 1 \mathrm{H}), 7.83$ $(\mathrm{d}, J=2.0 \mathrm{~Hz}, 1 \mathrm{H}), 7.73 \sim 7.59(\mathrm{~m}, 3 \mathrm{H}), 7.57 \sim 7.30(\mathrm{~m}$, $18 \mathrm{H}), 7.19$ (d, $J=8.8 \mathrm{~Hz}, 1 \mathrm{H}), 6.96(\mathrm{~d}, J=2.0 \mathrm{~Hz}, 1 \mathrm{H})$, $6.73(\mathrm{~d}, J=2.0 \mathrm{~Hz}, 1 \mathrm{H}), 5.34$ (d, $J=5.2 \mathrm{~Hz}, 1 \mathrm{H}), 5.30 \sim$ $5.07(\mathrm{~m}, 10 \mathrm{H}), 4.59(\mathrm{~d}, J=6.4 \mathrm{~Hz}, 1 \mathrm{H}), 4.33 \sim 4.31(\mathrm{~m}$, $2 \mathrm{H}), 3.94 \sim 3.71(\mathrm{~m}, 3 \mathrm{H}), 3.70(\mathrm{~s}, 3 \mathrm{H}), 3.67 \sim 3.54(\mathrm{~m}$, 2H), 2.99 (dd, $J=12.8,4.0 \mathrm{~Hz}, 1 \mathrm{H}), 1.97 \sim 1.92(\mathrm{~m}, 1 \mathrm{H})$, $1.89(\mathrm{~s}, 3 \mathrm{H}) ;{ }^{13} \mathrm{C}$ NMR (101 MHz, DMSO- $\left.d_{6}\right) \delta: 172.7$, $171.5,167.3,163.2,159.6,158.6,152.6,150.7,148.1$, $143.2,139.0,137.6,137.3,137.2$, 136.5, 129.0, 128.92, $128.88,128.85,128.68,128.51,128.37,128.27,128.12$, $128.05,128.0,127.5,123.2,122.2,114.6,114.0,109.3$, $98.3,94.7,89.6,75.4,70.7,70.5,70.4,69.0,66.6,64.3$, 64.1, 53.8, 52.1, 23.3; MS (ESI) $\mathrm{m} / z: 1049.3[\mathrm{M}+\mathrm{H}]^{+}$; HRMS (ESI) calcd for $\mathrm{C}_{58} \mathrm{H}_{57} \mathrm{~N}_{4} \mathrm{O}_{15}[\mathrm{M}+\mathrm{H}]^{+}$1049.3815, found 1049.3819 .

3.2.4 1- $[N-1-(\alpha-N$-乙酰神经氨酸 $)]-4-[3-O-$ - 亚甲基槲皮素]-[1,2,3]-三唑 $(\mathbf{2 0})$ 的合成

将化合物 12 (200 mg, $0.19 \mathrm{mmoL})$ 溶于 $10 \mathrm{~mL}$ 甲醇, 加入 $10 \% \mathrm{Pd} / \mathrm{C}(40 \mathrm{mg})$, 通入氢气，室温搅拌 $12 \mathrm{~h}$, 滤除 钯碳, 用 $2 \mathrm{~mol} \cdot \mathrm{L}^{-1}$ 氢氧化钾水溶液调 $\mathrm{pH}$ 至 $9 \sim 10$, 继 续搅拌 $2 \mathrm{~h}$, 用阳离子树脂调 $\mathrm{pH}$ 至中性, 过滤, 浓缩滤 液, 柱层析分离纯化 [洗脱剂: $V$ (乙酸乙酯)： $V$ (甲醇) : $V($ 水 $)=10: 1: 0.1)$ ]得黄色固体 $20105 \mathrm{mg}$, 产率 $82 \%$. m.p. $152.7 \sim 152.8{ }^{\circ} \mathrm{C} ;{ }^{1} \mathrm{H}$ NMR (400 MHz, MeOD) $\delta$ : 8.16 (s, 1H), 7.47 (s, 1H), 7.37 (d, $J=8.0 \mathrm{~Hz}, 1 \mathrm{H}), 6.84$ (d, $J=8.4 \mathrm{~Hz}, 1 \mathrm{H}), 6.35$ (s, 1H), 6.19 (s, 1H), 5.11 (s, 2H), $4.10 \sim 3.77(\mathrm{~m}, 5 \mathrm{H}), 3.77 \sim 3.54(\mathrm{~m}, 2 \mathrm{H}), 3.41 \sim 3.21(\mathrm{~m}$, 1H), $2.11 \sim 2.05(\mathrm{~m}, 4 \mathrm{H}) ;{ }^{13} \mathrm{C}$ NMR $(101 \mathrm{MHz}, \mathrm{MeOD}) \delta$ : $179.8,175.4,165.8,163.0,159.0,158.4,149.6,145.9$, 
137.2, 124.2, 122.8, 122.7, 117.0, 116.4, 105.9, 99.8, 94.8, 76.3, 72.4, 70.0, 68.8, 65.6, 64.5, 53.5, 41.1, 22.7; HRMS (ESI) calcd for $\mathrm{C}_{29} \mathrm{H}_{29} \mathrm{O}_{15} \mathrm{~N}_{4}[\mathrm{M}-\mathrm{H}]^{-}$673.1635, found 673.1627.

3.2.5 1- $[N-1-(\beta-D$-甘露糖 $)]-4-\left[3-O\right.$ - 亚甲基- $\left(5,7,3^{\prime}, 4^{\prime}-\right.$ 四苄氧基槲皮素)]-[1,2,3]-三唑(13)的合成

化合物 13 的合成方法同化合物 12, 以化合物 5 为 原料得浅黄色固体, 产率 91\%. m.p. 190.9 191.8 ${ }^{\circ} \mathrm{C}$; ${ }^{1} \mathrm{H}$ NMR (400 MHz, DMSO- $\left.d_{6}\right) \delta: 8.34(\mathrm{~s}, 1 \mathrm{H}), 7.87$ (d, $J=2.0 \mathrm{~Hz}, 1 \mathrm{H}), 7.72(\mathrm{dd}, J=8.4 \mathrm{~Hz}, 2.0 \mathrm{~Hz}, 1 \mathrm{H}), 7.65$ (d, $J=7.6 \mathrm{~Hz}, 2 \mathrm{H}), 7.53 \sim 7.32(\mathrm{~m}, 18 \mathrm{H}), 7.23(\mathrm{~d}, J=8.8 \mathrm{~Hz}$, $1 \mathrm{H}), 6.96(\mathrm{~d}, J=2.0 \mathrm{~Hz}, 1 \mathrm{H}), 6.73$ (d, $J=2.0 \mathrm{~Hz}, 1 \mathrm{H}), 6.00$ $(\mathrm{s}, 1 \mathrm{H}), 5.28 \sim 5.26(\mathrm{~m}, 7 \mathrm{H}), 5.19(\mathrm{~s}, 2 \mathrm{H}), 5.16(\mathrm{~s}, 2 \mathrm{H})$, $5.06(\mathrm{~d}, J=5.6 \mathrm{~Hz}, 1 \mathrm{H}), 5.00(\mathrm{~d}, J=5.6 \mathrm{~Hz}, 1 \mathrm{H}), 4.63(\mathrm{t}$, $J=6.0 \mathrm{~Hz}, 1 \mathrm{H}), 3.89 \sim 3.87(\mathrm{~m}, 1 \mathrm{H}), 3.74 \sim 3.72(\mathrm{~m}, 1 \mathrm{H})$, $3.62 \sim 3.60(\mathrm{~m}, 1 \mathrm{H}), 3.55 \sim 3.40(\mathrm{~m}, 3 \mathrm{H}) ;{ }^{13} \mathrm{C}$ NMR $(101$ MHz, DMSO- $\left.d_{6}\right) \delta: 172.8,163.2,159.6,158.6,152.5$, $150.7,148.2,139.4,137.6,137.3,137.2,136.5,129.0$, $128.9,128.8,128.7,128.5,128.4,128.3,128.1,128.1$, $128.0,127.4,124.9,123.3,122.3,114.4,114.1,109.4$, 98.3, 94.7, 86.4, 80.8, 73.6, 71.0, 70.7, 70.6, 70.5, 70.4, 66.7, 64.7, 61.5; MS (ESI) $m / z: 906.3[\mathrm{M}+\mathrm{H}]^{+}$; HRMS (ESI) calcd for $\mathrm{C}_{52} \mathrm{H}_{48} \mathrm{~N}_{3} \mathrm{O}_{12}[\mathrm{M}+\mathrm{H}]^{+}$906.3233, found 906.3227.

3.2.6 1-[N-1- $(\beta-D$ - 甘露糖 $)]-4-[3-O-$ 亚甲基 - 槲皮 素]-[1,2,3]-三唑(21) 的合成

将化合物 13 (200 mg, $0.22 \mathrm{mmoL})$ 溶于 $10 \mathrm{~mL}$ 甲醇, 加入 $10 \% \mathrm{Pd} / \mathrm{C}(40 \mathrm{mg})$, 通入氢气, 室温搅拌 $12 \mathrm{~h}$, 滤除 钯碳, 浓缩滤液, 柱层析分离纯化 [洗脱剂: $V($ 乙酸乙 酯) $: V$ (甲醇) $: V$ (水) $=30 ： 1 ： 0.1)$ ]得黄色固体 21114 mg, 产率 95\%. m.p. $175.8 \sim 179.5{ }^{\circ} \mathrm{C} ;{ }^{1} \mathrm{H}$ NMR (400 MHz, DMSO- $\left.d_{6}\right) \delta: 8.31(\mathrm{~s}, 1 \mathrm{H}), 7.51 \sim 7.47(\mathrm{~m}, 2 \mathrm{H}), 6.87$ $(\mathrm{d}, J=8.4 \mathrm{~Hz}, 1 \mathrm{H}), 6.42(\mathrm{~d}, J=2.0 \mathrm{~Hz}, 1 \mathrm{H}), 6.22$ (d, $J=$ $2.0 \mathrm{~Hz}, 1 \mathrm{H}), 6.00(\mathrm{~s}, 1 \mathrm{H}), 5.28(\mathrm{~s}, 1 \mathrm{H}), 5.11(\mathrm{~s}, 2 \mathrm{H}), 3.89 \sim$ $3.42(\mathrm{~m}, 6 \mathrm{H}) ;{ }^{13} \mathrm{C}$ NMR (101 MHz, DMSO- $\left.d_{6}\right) \delta: 178.3$, $164.6,161.7,156.8,156.7,149.1,145.6,142.7,136.6$, 125.0, 121.6, 121.2, 116.1, 115.8, 104.6, 99.1, 94.1, 86.3, 80.8, 73.6, 70.9, 66.7, 65.2, 61.5; HRMS (ESI) calcd for $\mathrm{C}_{24} \mathrm{H}_{22} \mathrm{O}_{12} \mathrm{~N}_{3}[\mathrm{M}-\mathrm{H}]^{-}$544.1209, found 544.1194.

3.2 .71 - $[N-1-(\beta-D$ - 葡萄糖 $)]-4-\left[3-O\right.$ - 亚甲基- $\left(5,7,3^{\prime}, 4^{\prime}-\right.$ 四芳氧基槲皮素)]-[1,2,3]-三唑(14)的合成

化合物 14 的合成方法同化合物 12, 以化合物 6 为 原料得黄色固体, 产率 $89 \%$. m.p. $121.7 \sim 122.1{ }^{\circ} \mathrm{C} ;{ }^{1} \mathrm{H}$ NMR (400 MHz, DMSO-d $\left.)_{6}\right) \delta: 8.39$ (s, 1H), 7.89 (s, 1H), $7.73(\mathrm{~d}, J=8.4 \mathrm{~Hz}, 1 \mathrm{H}), 7.65(\mathrm{~d}, J=7.2 \mathrm{~Hz}, 2 \mathrm{H}), 7.51 \sim$ $7.33(\mathrm{~m}, 18 \mathrm{H}), 7.24(\mathrm{~d}, J=8.4 \mathrm{~Hz}, 1 \mathrm{H}), 6.96(\mathrm{~s}, 1 \mathrm{H}), 6.74$ (s, 1H), 5.57 (d, $J=9.2 \mathrm{~Hz}, 1 \mathrm{H}), 5.39$ (d, $J=6.0 \mathrm{~Hz}, 1 \mathrm{H})$, $5.30 \sim 5.17(\mathrm{~m}, 11 \mathrm{H}), 4.64(\mathrm{~s}, 1 \mathrm{H}), 3.81 \sim 3.68(\mathrm{~m}, 2 \mathrm{H})$, $3.47 \sim 3.25(\mathrm{~m}, 4 \mathrm{H}) ;{ }^{13} \mathrm{C}$ NMR (101 MHz, DMSO- $\left.d_{6}\right) \delta$ : $172.8,163.2,159.6,158.6,152.5,150.7,148.2,143.2$, $139.4,137.7,137.3,137.2,136.5,129.0,128.9,128.8$, $128.7,128.6,128.4,128.3,128.1,128.0,127.4,124.4$, $123.2,122.4,114.4,114.1,109.3,98.3,94.7,88.0,80.5$, $77.4,72.6,70.7,70.6,70.5,70.3,70.0,64.5,61.2$; MS (ESI) $m / z: 906.3 \quad[\mathrm{M}+\mathrm{H}]^{+}$; HRMS (ESI) calcd for $\mathrm{C}_{52} \mathrm{H}_{48} \mathrm{~N}_{3} \mathrm{O}_{12}[\mathrm{M}+\mathrm{H}]^{+}$906.3233, found 906.3231.

$3.2 .81-[N-1-(\beta-D-$ 葡萄糖 $)]-4-[3-O-$ 亚甲基 - 槲皮 素]-[1,2,3]-三唑(22) 的合成

化合物 22 的合成方法同化合物 $\mathbf{2 1}$, 以化合物 14 为 原料得黄色固体，产率 93\%. m.p. 182.6 183.4 ${ }^{\circ} \mathrm{C} ;{ }^{1} \mathrm{H}$ NMR (400 MHz, DMSO- $\left.d_{6}\right) \delta: 8.35(\mathrm{~s}, 1 \mathrm{H}), 7.52$ (d, $J=$ $8.4 \mathrm{~Hz}, 1 \mathrm{H}), 7.51$ (s, 1H), 6.89 (d, $J=8.4 \mathrm{~Hz}, 1 \mathrm{H}), 6.42$ (s, $1 \mathrm{H}), 6.22(\mathrm{~s}, 1 \mathrm{H}), 5.55(\mathrm{~d}, J=9.2 \mathrm{~Hz}, 1 \mathrm{H}), 5.12(\mathrm{~s}, 2 \mathrm{H})$, $4.07 \sim 3.12(\mathrm{~m}, 6 \mathrm{H}) ;{ }^{13} \mathrm{C}$ NMR $\left(101 \mathrm{MHz}, \mathrm{DMSO}-d_{6}\right) \delta$ : $178.3,164.7,161.8,156.8,156.7,149.2,145.7,143.1$, $136.6,124.5,12.6,121.2,116.2,115.8,104.6,99.1,94.1$, 88.0, 80.5, 77.5, 72.5, 70.0, 65.2, 61.3; HRMS (ESI) calcd for $\mathrm{C}_{24} \mathrm{H}_{22} \mathrm{O}_{12} \mathrm{~N}_{3}[\mathrm{M}-\mathrm{H}]^{-}$544.1209, found 544.1216.

3.2.9 1-[N-1-( $\beta$ - $D$-半乳糖 $)]-4$-[3- $O$ - 亚甲基- $\left(5,7,3^{\prime}, 4^{\prime}-\right.$ 四苄氧基槲皮素)]-[1,2,3]-三唑(15)的合成

化合物 15 的合成方法同化合物 12 , 以化合物 7 为 原料得黄色固体, 产率 $92 \%$. m.p. 129.9 $130.2{ }^{\circ} \mathrm{C} ;{ }^{1} \mathrm{H}$ NMR (400 MHz, DMSO- $\left.d_{6}\right) \delta: 8.35(\mathrm{~s}, 1 \mathrm{H}), 7.88(\mathrm{~s}, 1 \mathrm{H})$, $7.72(\mathrm{~d}, J=8.8 \mathrm{~Hz}, 1 \mathrm{H}), 7.65$ (d, $J=7.6 \mathrm{~Hz}, 2 \mathrm{H}), 7.51 \sim$ $7.33(\mathrm{~m}, 18 \mathrm{H}), 7.23$ (d, J=8.8 Hz, 1H), 6.97 (s, 1H), 6.74 $(\mathrm{s}, 1 \mathrm{H}), 5.51(\mathrm{~d}, J=8.8 \mathrm{~Hz}, 1 \mathrm{H}), 5.28 \sim 5.12(\mathrm{~m}, 8 \mathrm{H}), 5.17$ $(\mathrm{s}, 2 \mathrm{H}), 5.01(\mathrm{~d}, J=5.6 \mathrm{~Hz}, 1 \mathrm{H}), 4.71(\mathrm{t}, J=5.6 \mathrm{~Hz}, 1 \mathrm{H})$, $4.63(\mathrm{~d}, J=5.6 \mathrm{~Hz}, 1 \mathrm{H}), 4.07 \sim 4.02(\mathrm{~m}, 1 \mathrm{H}), 3.79 \sim 3.49$ $(\mathrm{m}, 5 \mathrm{H}) ;{ }^{13} \mathrm{C}$ NMR $\left(101 \mathrm{MHz}, \mathrm{DMSO}-d_{6}\right) \delta: 172.8,163.2$, $159.6,158.6,152.4,150.7,148.2,139.4,137.7,137.3$, $137.2,136.5,129.0,128.9,128.8,128.7,128.5,128.4$, $128.3,128.1,128.0,127.4,124.1,123.3,122.3,114.5$, $114.1,109.4,98.3,94.7,88.6,78.9,74.2,70.7,70.6,70.5$, 70.4, 69.9, 68.9, 64.6, 60.8; MS (ESI) $m / z: 906.3[\mathrm{M}+$ $\mathrm{H}]^{+}$; HRMS (ESI) calcd for $\mathrm{C}_{52} \mathrm{H}_{48} \mathrm{~N}_{3} \mathrm{O}_{12}[\mathrm{M}+\mathrm{H}]^{+}$ 906.3233, found 906.3231 .

3.2.10 1-[N-1- $(\beta-D$ - 半乳糖 $)]-4-[3-O$ - 亚甲基-槲皮 素]-[1,2,3]-三唑(23) 的合成

化合物 23 的合成方法同化合物 $\mathbf{2 1}$, 以化合物 15 为 原料得浅黄色固体, 产率 $96 \%$. m.p. $110.0 \sim 110.2{ }^{\circ} \mathrm{C}$; 
${ }^{1} \mathrm{H}$ NMR (400 MHz, DMSO- $\left.d_{6}\right) \delta: 8.35(\mathrm{~s}, 1 \mathrm{H}), 7.51(\mathrm{~d}$, $J=8.8 \mathrm{~Hz}, 1 \mathrm{H}), 7.50$ (s, $1 \mathrm{H}), 6,88(\mathrm{~d}, J=8.8 \mathrm{~Hz}, 1 \mathrm{H}), 6.43$ (d, $J=2.0 \mathrm{~Hz}, 1 \mathrm{H}), 6.22(\mathrm{~d}, J=2.0 \mathrm{~Hz}, 1 \mathrm{H}), 5.50$ (d, $J=$ $9.2 \mathrm{~Hz}, 1 \mathrm{H}), 5.12(\mathrm{~s}, 2 \mathrm{H}), 4.10 \sim 4.02(\mathrm{~m}, 1 \mathrm{H}), 3.82 \sim 3.54$ $(\mathrm{m}, 5 \mathrm{H}) ;{ }^{13} \mathrm{C}$ NMR (101 MHz, DMSO- $\left.d_{6}\right) \delta: 178.3,164.6$, $161.8,156.8,156.6,149.2,145.7,143.2,136.7,124.2$, 121.6, 121.2, 116.2, 115.8, 104.7, 99.1, 94.1, 88.6, 78.9, 74.2, 69.8, 69.0, 65.2, 60.9; HRMS (ESI) calcd for $\mathrm{C}_{24} \mathrm{H}_{22} \mathrm{O}_{12} \mathrm{~N}_{3}[\mathrm{M}-\mathrm{H}]^{-}$544.1209, found 544.1184.

3.2.11 1-[N-1-( $\beta-D$ - 吡喃半乳糖基- $(1 \rightarrow 4)-D$ - 吡喃茂 萄糖)]-4-[3- $O$ - 亚甲基-(5,7, $3^{\prime}, 4^{\prime}$ - 四芳氧基藓皮 素)]-[1,2,3]-三唑(16)的合成

化合物 16 的合成方法同化合物 12, 以化合物 8 为 原料得黄色固体, 产率 $81 \%$. m.p. $108.1{ }^{\circ} \mathrm{C} ;{ }^{1} \mathrm{H}$ NMR $\left(400 \mathrm{MHz}, \mathrm{DMSO}-d_{6}\right) \delta: 8.40(\mathrm{~s}, 1 \mathrm{H}), 7.89(\mathrm{~d}, J=2.0 \mathrm{~Hz}$, $1 \mathrm{H}), 7.73(\mathrm{~d}, J=8.4 \mathrm{~Hz}, 1 \mathrm{H}), 7.65(\mathrm{~d}, J=7.2 \mathrm{~Hz}, 2 \mathrm{H})$, $7.54 \sim 7.32(\mathrm{~m}, 18 \mathrm{H}), 7.23(\mathrm{~d}, J=8.4 \mathrm{~Hz}, 1 \mathrm{H}), 6.96$ (d, $J=$ $2.0 \mathrm{~Hz}, 1 \mathrm{H}), 6.73(\mathrm{~d}, J=2.0 \mathrm{~Hz}, 1 \mathrm{H}), 5.68(\mathrm{~d}, J=9.2 \mathrm{~Hz}$, $1 \mathrm{H}), 5.59(\mathrm{~d}, J=6.0 \mathrm{~Hz}, 1 \mathrm{H}), 5.27 \sim 5.14(\mathrm{~m}, 11 \mathrm{H}), 4.91$ (s, 1H), $4.82(\mathrm{~d}, J=4.8 \mathrm{~Hz}, 1 \mathrm{H}), 4.70 \sim 4.67(\mathrm{~m}, 2 \mathrm{H}), 4.56$ (d, $J=4.8 \mathrm{~Hz}, 1 \mathrm{H}), 4.27$ (d, $J=7.2 \mathrm{~Hz}, 1 \mathrm{H}), 3.88$ (dd, $J=$ $15.2,9.2 \mathrm{~Hz}, 1 \mathrm{H}), 3.79 \sim 3.75(\mathrm{~m}, 1 \mathrm{H}), 3.66 \sim 3.50(\mathrm{~m}$, $8 \mathrm{H}) ;{ }^{13} \mathrm{C}$ NMR (101 MHz, DMSO) $\delta: 172.8,163.2,159.6$, $158.6,152.5,150.7,148.2,139.4,137.6,137.3,137.2$, $136.5,129.0,128.9,128.9,128.8,128.7,128.5,128.4$, $128.3,128.1,128.1,127.4,124.4,123.3,122.4,114.5$, 114.1, 109.3, 104.3, 98.3, 94.7, 87.5, 80.3, 78.3, 76.1, 75.7, 73.7, 72.3, 71.0, 70.8, 70.6, 70.5, 70.4, 68.6, 64.5, 60.9; MS (ESI) $m / z: 1068.4[\mathrm{M}+\mathrm{H}]^{+}$; HRMS (ESI) calcd for $\mathrm{C}_{58} \mathrm{H}_{58} \mathrm{~N}_{3} \mathrm{O}_{17}[\mathrm{M}+\mathrm{H}]^{+}$1068.3761, found 1068.3759.

3.2.12 1-[N-1- $(\beta-D$ - 吡喃半乳糖基 $-(1 \rightarrow 4)-D$ - 吡喃葡 萄糖)]-4-[3- $O$-亚甲基-檞皮素]-[1,2,3]-三唑(24)的合 成

化合物 $\mathbf{2 4}$ 的合成方法同化合物 $\mathbf{2 1}$, 以化合物 $\mathbf{1 6}$ 为 原料得浅黄色固体，产率 91\%. m.p. 103.4 103.6 ${ }^{\circ} \mathrm{C}$; ${ }^{1} \mathrm{H}$ NMR (400 MHz, DMSO- $d_{6}$ ) $\delta: 8.39(\mathrm{~s}, 1 \mathrm{H}), 7.53(\mathrm{~d}$, $J=8.0 \mathrm{~Hz}, 1 \mathrm{H}), 7.53(\mathrm{~s}, 1 \mathrm{H}), 6.90(\mathrm{~d}, J=8.0 \mathrm{~Hz}, 1 \mathrm{H}), 6.44$ (s, 1H), $6.23(\mathrm{~s}, 1 \mathrm{H}), 5.67(\mathrm{~d}, J=9.2 \mathrm{~Hz}, 1 \mathrm{H}), 5.13(\mathrm{~s}, 2 \mathrm{H})$, $4.28(\mathrm{~d}, J=7.2 \mathrm{~Hz}, 1 \mathrm{H}), 3.98 \sim 3.44(\mathrm{~m}, 12 \mathrm{H}) ;{ }^{13} \mathrm{C}$ NMR $\left(101 \mathrm{MHz}, \mathrm{DMSO}-d_{6}\right) \delta: 178.3,164.7,161.7,156.8,156.7$, $149.2,145.7,143.2,136.6,124.5,121.6,121.2,116.2$, 115.8, 104.6, 104.3, 99.1, 94.1, 87.5, 80.3, 78.3, 76.1, 75.7, 73.7, 72.3, 71.0, 68.6, 65.1, 60.9, 60.5; HRMS (ESI) calcd for $\mathrm{C}_{30} \mathrm{H}_{32} \mathrm{O}_{17} \mathrm{~N}_{3}[\mathrm{M}-\mathrm{H}]^{-}$706.1737, found 706.1744.
3.2.13 1-[N-1-(3,6,9-三氧杂-十一-1-醇) ]-4-[3- $O$ - 亚 甲基-(5,7, $3^{\prime}, 4^{\prime}$ - 四芳氧基檞皮素)]-[1,2,3]-三唑(17) 的 合成

化合物 17 的合成方法同化合物 $\mathbf{1 2}$, 以化合物 9 为 原料得白色固体, 产率 $92 \%$. m.p. $109.3 \sim 109.5{ }^{\circ} \mathrm{C} ;{ }^{1} \mathrm{H}$ NMR (400 MHz, MeOD) $\delta: 7.88(\mathrm{~s}, 1 \mathrm{H}), 7.43(\mathrm{~s}, 1 \mathrm{H})$, 7.37 (d, $J=8.4 \mathrm{~Hz}, 1 \mathrm{H}), 7.36 \sim 6.96(\mathrm{~m}, 20 \mathrm{H}), 6.80(\mathrm{~d}, J=$ $8.4 \mathrm{~Hz}, 1 \mathrm{H}), 6.29$ (s, 1H), 6.14 (s, 1H), 5.14 (s, 2H), 4.42 (t, $J=5.6 \mathrm{~Hz}, 2 \mathrm{H}), 3.73(\mathrm{t}, J=5.6 \mathrm{~Hz}, 2 \mathrm{H}), 3.59 \sim 3.49(\mathrm{~m}$, 12H); MS (ESI) $m / z: 920.4[\mathrm{M}+\mathrm{H}]^{+}$; HRMS (ESI) calcd for $\mathrm{C}_{54} \mathrm{H}_{54} \mathrm{~N}_{3} \mathrm{O}_{11}[\mathrm{M}+\mathrm{H}]^{+}$920.3753, found 920.3755 .

3.2.14 1-[N-1-(3,6,9-三氧杂-十一-1-醇) ]-4-[3- $O$-亚 甲基-檞皮素]-[1,2,3]-三唑(25)的合成

化合物 $\mathbf{2 5}$ 的合成方法同化合物 $\mathbf{2 1}$, 以化合物 $\mathbf{1 7}$ 为 原料得浅黄色固体，产率 $92 \%$. m.p. $135.7 \sim 138.3{ }^{\circ} \mathrm{C}$; ${ }^{1} \mathrm{H}$ NMR (400 MHz, MeOD) $\delta: 7.88$ (s, 1H), $7.43(\mathrm{~s}, 1 \mathrm{H})$, 7.35 (d, $J=8.4 \mathrm{~Hz}, 1 \mathrm{H}), 6.80$ (d, $J=8.4 \mathrm{~Hz}, 1 \mathrm{H}), 6.29$ (s, $1 \mathrm{H}), 6.14(\mathrm{~s}, 1 \mathrm{H}), 5.13(\mathrm{~s}, 2 \mathrm{H}), 4.44(\mathrm{t}, J=5.2 \mathrm{~Hz}, 2 \mathrm{H})$, $3.75(\mathrm{t}, J=5.2 \mathrm{~Hz}, 2 \mathrm{H}), 3.63 \sim 3.49(\mathrm{~m}, 12 \mathrm{H}) ;{ }^{13} \mathrm{C}$ NMR (101 MHz, MeOD) $\delta$ : 178.4, 164.3, 161.6, 157.3, 156.9, $148.3,144.7,142.8,135.8,125.6,121.6,121.4,115.4$, $114.9,104.5,98.4,93.4,72.2,70.1,70.0,69.9,68.8,64.0$, 60.8, 49.9; HRMS (ESI) calcd for $\mathrm{C}_{26} \mathrm{H}_{29} \mathrm{O}_{11} \mathrm{~N}_{3} \mathrm{Na}[\mathrm{M}+$ $\mathrm{Na}]^{+}$582.1694, found, 582.1676.

3.2.15 1-[N-1-(聚乙二醇单甲醚 2000)]-4-[3- $O$ - 亚甲 基-(5,7,3', $4^{\prime}$-四芳氧基檞皮素)]-[1,2,3]-三唑(18)的合成

化合物 $\mathbf{1 8}$ 的合成方法同化合物 $\mathbf{1 2}$, 以化合物 $\mathbf{1 0}$ 为 原料得白色固体，产率 $89 \%$. m.p. 113.2 113.3 ${ }^{\circ} \mathrm{C}$; MALDI-TOF-MS calcd for $\mathrm{C}_{137} \mathrm{H}_{220} \mathrm{O}_{52} \mathrm{~N}_{3}[\mathrm{M}+\mathrm{H}]^{+}$ 2740.4691, found 2740.4682. 粗产品未经分离纯化直接 用于下一步反应.

3.2.16 1-[N-1-(聚乙二醇单甲醚 2000)]-4-[3- $O$ - 亚甲 基-檞皮素]-[1,2,3]-三唑(26)的合成

化合物 $\mathbf{2 6}$ 的合成方法同化合物 $\mathbf{2 1}$, 以化合物 $\mathbf{1 8}$ 为 原料得浅黄色固体，产率 $93 \%$. m.p. $81.2 \sim 81.4{ }^{\circ} \mathrm{C} ;{ }^{1} \mathrm{H}$ NMR (400 MHz, MeOD) $\delta: 7.95(\mathrm{~s}, 1 \mathrm{H}), 7.47(\mathrm{~s}, 1 \mathrm{H})$, $7.42(\mathrm{~d}, J=8.4 \mathrm{~Hz}, 1 \mathrm{H}), 6.84(\mathrm{~d}, J=8.4 \mathrm{~Hz}, 1 \mathrm{H}), 6.39$ (d, $J=2.0 \mathrm{~Hz}, 1 \mathrm{H}), 6.21(\mathrm{~d}, J=2.0 \mathrm{~Hz}, 1 \mathrm{H}), 5.20(\mathrm{~s}, 2 \mathrm{H}), 4.48$ (t, $J=5.2 \mathrm{~Hz}, 2 \mathrm{H}), 3.78(\mathrm{t}, J=5.2 \mathrm{~Hz}, 3 \mathrm{H}), 3.63 \sim 3.35(\mathrm{~m}$, 174H), 3.31 3.30 (m, 4H); ${ }^{13} \mathrm{C}$ NMR (101 MHz, MeOD) $\delta: 179.9,165.9,163.2,158.7,158.4,149.8,146.2,144.2$, $137.2,127.0,123.0,122.8,116.8,116.3,106.0,99.8,94.8$, 73.0, 71.6, 71.5, 71.4, 70.2, 65.5, 59.1, 51.4; MALDI-TOF-MS calcd for $\mathrm{C}_{137} \mathrm{H}_{220} \mathrm{O}_{57} \mathrm{~N}_{3} \quad[\mathrm{M}+\mathrm{H}]$ 2380.2818, found 2380.2822 . 
3.2.17 1-[ $N$-1-(1-( $\beta$ - $D$-半乳糖) - $3,6,9$-三氧杂-十一烷 基]-4-[3- $O$ - 亚甲基 - $\left(5,7,3^{\prime}, 4^{\prime}\right.$ - 四芐氧基解皮 素)]-[1,2,3]-三唑(19)的合成

化合物 19 的合成方法同化合物 $\mathbf{1 2}$, 以化合物 11 为 原料得浅黄色固体, 产率 82\%. m.p. $102.1{ }^{\circ} \mathrm{C} ;{ }^{1} \mathrm{H}$ NMR $\left(400 \mathrm{MHz}, \mathrm{DMSO}-d_{6}\right) \delta: 8.34(\mathrm{~s}, 1 \mathrm{H}), 7.52(\mathrm{~s}, 1 \mathrm{H}), 7.50$ (d, $J=8.4 \mathrm{~Hz}, 1 \mathrm{H}), 7.36 \sim 6.96(\mathrm{~m}, 20 \mathrm{H}), 6.83(\mathrm{~d}, J=8.4$ $\mathrm{Hz}, 1 \mathrm{H}), 6.33$ (s, 1H), 6.25 (s, 1H), $5.14(\mathrm{~s}, 2 \mathrm{H}), 4.48(\mathrm{t}$, $J=4.40 \mathrm{~Hz}, 2 \mathrm{H}), 4.26(\mathrm{~d}, J=7.2 \mathrm{~Hz}, 1 \mathrm{H}), 4.01 \sim 3.97(\mathrm{~m}$, $1 \mathrm{H}), 3.87(\mathrm{~d}, J=2.4 \mathrm{~Hz}, 1 \mathrm{H}), 3.79 \sim 3.50(\mathrm{~m}, 18 \mathrm{H})$; MS (ESI) $m / z:[\mathrm{M}+\mathrm{H}]^{+}$1082.4; HRMS (ESI) calcd for $\mathrm{C}_{60} \mathrm{H}_{64} \mathrm{~N}_{3} \mathrm{O}_{16}[\mathrm{M}+\mathrm{H}]^{+}$1082.4281, found 1082.4279.

3.2 .18 1-[N-1-((1-( $\beta-D$-半乳糖) - 3, 6,9-三氧杂-十一烷 基)]-4-[3- $O$-亚甲基-檞皮素]-[1,2,3]-三唑(27)的合成

化合物 $\mathbf{2 7}$ 的合成方法同化合物 $\mathbf{2 1}$, 以化合物 19 为 原料得浅黄色固体, 产率 $90 \%$. m.p. $130.4 \sim 132.0{ }^{\circ} \mathrm{C}$; ${ }^{1} \mathrm{H}$ NMR (400 MHz, MeOD) $\delta$ : 7.91 (s, 1H), $7.45(\mathrm{~s}, 1 \mathrm{H})$, 7.37 (d, $J=8.4 \mathrm{~Hz}, 1 \mathrm{H}), 6.83$ (d, $J=8.4 \mathrm{~Hz}, 1 \mathrm{H}), 6.30$ (s, $1 \mathrm{H}), 6.15(\mathrm{~s}, 1 \mathrm{H}), 5.14(\mathrm{~s}, 2 \mathrm{H}), 4.48$ (t, $J=4.0 \mathrm{~Hz}, 2 \mathrm{H})$, $4.26(\mathrm{~d}, J=7.2 \mathrm{~Hz}, 1 \mathrm{H}), 4.01 \sim 3.97(\mathrm{~m}, 1 \mathrm{H}), 3.87$ (d, $J=$ $2.4 \mathrm{~Hz}, 1 \mathrm{H}), 3.79 \sim 3.50(\mathrm{~m}, 18 \mathrm{H}) ;{ }^{13} \mathrm{C}$ NMR $(101 \mathrm{MHz}$, MeOD) $\delta: 178.3,164.3,161.5,157.2,156.8,148.2,144.6$, $142.8,135.8,125.7,121.6,121.5,115.4,115.0,104.5$, $103.5,98.5,93.5,75.2,73.4,71.2,69.9,69.9,69.0,68.8$, 68.2, 64.1, 61.2, 49.9; HRMS (ESI) calcd for $\mathrm{C}_{32} \mathrm{H}_{38} \mathrm{O}_{16} \mathrm{~N}_{3}$ $[\mathrm{M}-\mathrm{H}]^{-} 720.2258$, found 720.2237 .

\section{3 生物活性测试}

在生理状态下, 检测了化合物 20 27 对 3T3-L1 脂 肪细胞葡萄糖消耗以及 $2-D$-脱氧葡萄糖摄取的影响. 以 二甲双胍作为阳性对照.

\subsection{1 脂肪细胞糖消耗的实验}

将分化成熟生长状态良好的脂肪细胞接种于 48 孔 培养板中 $\left(2 \times 10^{5} \mathrm{cell} /\right.$ 孔 $)$, 细胞生长至约 $80 \%$ 密度时, 将培养基换成不含糖的 KRHB 液同步化 $4 \mathrm{~h}$. 分组如下:

(1)空白组, 化合物组(DMSO 溶解, $10 \mu \mathrm{mol} \cdot \mathrm{L}^{-1}$ ), 和 Metformin $\left(1 \mathrm{mmol} \cdot \mathrm{L}^{-1}\right)$ 温育 $30 \mathrm{~min}$ 后, 再加入葡萄 糖(终浓度为 $11 \mathrm{mmol} \cdot \mathrm{L}^{-1}$ ) 后, 于 $37{ }^{\circ} \mathrm{C}$ 再睬育 $4 \mathrm{~h}$. (2)空 白组, 化合物组(PBS 溶解, $10 \mu \mathrm{mol} \cdot \mathrm{L}^{-1}$ ) 和 Metformin $\left(1 \mathrm{mmol} \cdot \mathrm{L}^{-1}\right)$ 温育 $30 \mathrm{~min}$ 后, 再加入葡萄糖(终浓度为 11 $\mathrm{mmol} \cdot \mathrm{L}^{-1}$ ) 后, 于 $37{ }^{\circ} \mathrm{C}$ 再捊育 $4 \mathrm{~h}$.

用葡萄糖测定试剂盒测定细胞上清液中葡萄糖的 含量, 通过计算葡萄糖浓度差值即为脂肪细胞葡萄糖的 消耗量.

\subsection{2 脂肪细胞 $2-D$-脱氧葡萄糖摄取的实验}

将分化成熟生长状态良好的脂肪细胞, 接种于 48 孔培养板中 $\left(2 \times 10^{5} \mathrm{cell} /\right.$ 孔 $)$, 细胞生长至约 $80 \%$ 密度 时, 将培养基换成不含糖的 KRHB 液同步化 $4 \mathrm{~h}$. 分组 如下：空白组, 化合物组( $\mathrm{PBS}$ 溶解, $10 \mu \mathrm{mol} \cdot \mathrm{L}^{-1}$ ) 和 Metformin $\left(1 \mathrm{mmol} \cdot \mathrm{L}^{-1}\right)$ 温育 $30 \mathrm{~min}$ 后, 再加入葡萄糖 (终浓度为 $11 \mathrm{mmol} \cdot \mathrm{L}^{-1}$ ) 后, 于 $37{ }^{\circ} \mathrm{C}$ 再孵育 $0.5 \mathrm{~h}$. 用 PBS 清洗后, 加 $100 \mu \mathrm{L}$ 的 2-D-脱氧葡萄糖(2-NBDG) $\left(500 \mu \mathrm{mol} \cdot \mathrm{L}^{-1}\right)$ 探针, 于 $37^{\circ} \mathrm{C}$ 避光孵育 $1 \mathrm{~h}$. 用冷的 $\mathrm{PBS}$ 洗涤 3 次, 每孔加入 $200 \mu \mathrm{L}$ 的 KRHB, 在 $480 \mathrm{~nm}$ 激发 波长和 $515 \mathrm{~nm}$ 发射波长下用苂光显微镜拍照.

辅助材料(Supporting Information) 中间体 3、目标产 物 $\mathbf{2 0} \sim \mathbf{2 7}$ 的 ${ }^{1} \mathrm{H}$ NMR 和 ${ }^{13} \mathrm{C}$ NMR 谱图. 这些材料可以 免费从本刊网站(http://sioc-journal.cn/)上下载.

\section{References}

[1] Seo, M. J.; Lee, Y. J.; Hwang, J. H.; Kim, K. J.; Lee, B. Y. J. Nutr. Biochem. 2015, 11, 1308.

[2] Kim, C. S.; Kwon, Y.; Choe, S. Y.; Hong, S. M.; Yoo, H.; Goto, T.; Kawada, T.; Choi, H. S.; Joe, Y.; Chung, H. T.; Yu, R. Nutr. Metab. (Lond) 2015, 12, 33.

[3] Mahmoud, M. F.; Hassan, N. A.; El Bassossy, H. M.; Fahmy, A. PLoS One 2013, 8, e63784.

[4] Hertog, M. G.; Bueno-de-Mesquita, H. B.; Fehily, A. M.; Sweetnam, P. M.; Elwood, P. C. Cancer Epidemiol. Biomarkers Prev. 1996, 5, 673.

[5] Shoskes, D. A.; Nickel, J. C. Urologic Clinics North Am. 2011, 38, 279.

[6] Liu, K.; Mei, F.; Wang, Y. P.; Xiao, N.; Yang, L. L.; Wang, Y. L.; Li, J.; Huang, F.; Kou, J. P.; Liu, B. L; Qi, L. W. Mol. Nutr. Food Res. 2016, 60, 551 .

[7] Bhadada, S. V.; Vyas, V. K.; Goyal, R. K. Biomed. Pharmacother. 2016, 83, 221.

[8] Casado-Díaz, A.; Anter, J.; Dorado, G.; Quesada-Gómez, J. M. J. Nutr. Biochem. 2016, 32, 151.

[9] Stefek, M.; Karasu, C. Rejuvenation Res. 2011, 14, 525.

[10] Mei, Q. G.; Yuan, W. C.; Wang, C. Chin. J. Org. Chem. 2015, 35, 70 (in Chinese) (梅青刚，袁伟成，王淳，有机化学, 2015, 35, 70.)

[11] Wang, Q. A.; Wu, Z.; Liu, L.; Zou, L. H.; Luo, M. Chin. J. Org. Chem. 2010, 30, 1682 (in Chinese). (汪秋安, 吴峥, 刘莉, 邹亮华, 罗茗, 有机化学, 2010, 30, 1682.)

[12] Yan, X.; Liu, H. Q.; Zou, Y. Q.; Ren, Z. H. Chin. J. Org. Chem. 2008, 28, 1534 (in Chinese). (延坌, 刘会青, 邹永青, 任占华, 有机化学, 2008, 28, 1534.)

[13] Teng, Y. O.; Zhao, H. Y.; Wang, J.; Liu, H.; Gao, M. L.; Zhou, Y.; Han, K. L.; Fan, Z. C.; Zhang, Y. M.; Sun, H.; Yu, P. Eur. J. Med. Chem. 2016, 112, 145.

[14] Sun, H.; Li, Y. S.; Zhang, X. T.; Lei, Y. N.; Ding, W. N.; Zhao, X.; Wang, H. M.; Song, X. T.; Yao, Q. W.; Zhang, Y. M.; Ma, Y.; Wang, R.; Zhu, T.; Yu, P. Bioorg. Med. Chem. Lett. 2015, 25, 4567.

[15] Lou, D. H.; Wang, G. Q.; Xia, L.; Chen, L.; Wang, Q. A. Chin. J. Org. Chem. 2013, 33, 535 (in Chinese). (娄定辉, 汪钢强, 夏立, 陈凌, 汪秋安, 有机化学, 2013, 33, 535.) 
[16] Zhao, Z. G.; Wang, X. H.; Shi, Z. C.; Cheng, Y. Y. Chin. J. Org. Chem. 2014, 34, 1110 (in Chinese).

(赵志刚, 王晓红, 石治川, 程玉宇, 有机化学, 2014, 34, 1110.)

[17] Xiao, N.; Mei, F.; Sun, Y.; Pan, G. J.; Liu, B. L.; Liu, K. Planta Med. 2014, 80, 993.

[18] Pan, G. J.; Zhao, L. B.; Xiao, N.; Yang, K.; Ma, Y. T.; Zhao, X.; Fan, Z. C.; Zhang, Y. M.; Yao, Q. W.; Lu, K.; Yu, P. Eur. J. Med. Chem. 2016, 122, 674.

[19] Weïwer, M.; Chen, C. C.; Kemp, M. M.; Linhardt, R. J. Eur. J. Org. Chem. 2009, 10.1002/ejoc.200900117.
[20] Hanessian, S.; Saavedra, O. M.; Mascitti, V.; Marterer, W.; Dehriein; R.; Mak, C. P. Tetrahedron 2001, 57, 3267.

[21] Tso, K. K.; Leung, K. K.; Liu, H. W.; Lo, K. K. Chem. Commun. 2016, 52, 4557.

[22] Michalina, P.; Florence, C. D.; Alain, M.; Stanisław, P. Carbohydr. Res. 2015, 414, 51.

[23] Zhang, S.; Moussodia, R. O.; Sun, H. J.; Leowanawat, P.; Muncan, A.; Nusbaum, C. D.; Chelling, K. M.; Heiney, P. A.; Klein, M. L.; André, S.; Roy, R.; Gabius, H. J.; Percec, V. Angew. Chem., Int. Ed. Engl. 2014, 53, 10899.

(Zhao, C.) 